\title{
High-Throughput Development of Polymorphic Simple Sequence Repeat Markers Using Two Whole Genome Sequence Data in Peucedanum japonicum
}

\author{
Junki Lee ${ }^{1 \dagger}$, Ho Jun $\mathrm{Joh}^{1 \dagger}$, Nam-Hoon Kim, Sang-Choon Lee ${ }^{1}$, Woojong Jang ${ }^{1}$, Beom Soon Choi ${ }^{2}$, Yeisoo Yu ${ }^{2}$, \\ Tae-Jin Yang ${ }^{1,3} *$ \\ ${ }^{1}$ Deptartment of Plant Science, Plant Genomics and Breeding Institute, and Research Institute of Agriculture and Life Sciences, \\ College of Agriculture and Life Sciences, Seoul National University, Seoul 08826, Korea \\ ${ }^{2}$ Phyzen Genomics Institute, Seongnam 13558, Korea \\ ${ }^{3}$ Crop Biotechnology Institute/GreenBio Science and Technology, Seoul National University, Pyeongchang 25354, Korea
}

\begin{abstract}
Resource plants are important and have strong potential for a variety of utilities as crops or pharmaceutical materials. However, most resource plants remain wild and thus their utility for breeding and biotechnology is limited. Molecular markers are useful to initiate genetic study and molecular breeding for these understudied resource plants. We collected various wild collections of Peucedanum japonicum which is indigenous resource plants utilized as oriental medicine and leafy vegetables in Korea. In this study, we produced two independent whole genome sequences (WGSs) from two collections and identified large scale polymorphic simple sequence repeat (pSSR) based on our pipeline to develop SSR markers based on comparison of two WGSs. We identified a total of 452 candidate pSSR contigs. To confirm the accuracy and utility of pSSR, we designed ten SSR primer pairs and successfully applied those to seven collections of $P$. japonicum. The WGS and pSSR candidates identified in this study will be useful resource for genetic research and breeding purpose for the valuable resource plant, $P$. japonicum.
\end{abstract}

Keywords Molecular markers, Polymorphisms, Simple Sequence Repeats, Whole Genome Sequence, Peucedanum japonicum

\section{INTRODUCTION}

Indigenous resource plants have important values for a variety of utilities such as crops or pharmaceutical materials although there is limited research. Molecular markers are actively applied in many major crops, but the development of marker on the minor crops and resource plants is still limited. A molecular marker is a particular DNA fragment which includes specific genetic information with genome- level polymorphisms (Agarwal et al. 2008). Molecular markers have been widely used as valuable tools to measure genetic variation and thereby highly enhanced the genetic analysis on plants (Varshney et al. 2005).

Simple sequence repeats (SSRs, otherwise called as microsatellites) (Hiroe et al. 1958; Jones et al. 2010) which consist of sequential repeat of one to six or more nucleotide motifs in a head-to-tail structure (Kelkar et al. 2010) have been used often in genetic studies. SSR markers are further universally implemented for intraspecies variation and population genetic studies such as parentage analysis, fingerprinting, genetic structure analysis and genetic mapping (Mittal et al. 2009; Meng et al. 2014; Grover et al. 2016) due to variable polymorphic features and high

Received May 13, 2017; Revised May 17, 2017; Accepted May 18, 2017; Published June 1, 2017

*Corresponding author Tae-Jin Yang, tjyang@snu.ac.kr, Tel: +82-2-880-4547, Fax: +82-2-873-2056

${ }^{\dagger}$ These authors contributed equally. 
reproducibility. Because of the utility and advantage of SSR markers, various programs have been developed to identify SSR motifs and primer design such as MISA (http://pgrc.ipk-gatersleben.de/misa/), SSR Locator, Primer3, and FullSSR (Rozen et al. 2000; da Maia et al. 2008; Abdelkrim et al. 2009; Untergasser et al. 2012; Metz et al. 2016). However, conventional SSR marker development and experimental verification still require long guided sequence information and a labor-intensive trial and error procedure by repetitive wet experiments to find polymorphic SSR markers (Ma et al. 2009).

Peucedanum japonicum is a perennial herb that belong to the Apiaceae family and grows on the cliff or between rocks in coastal regions of Taiwan, Japan, China and Korea (Buwalda 1949; Hiroe and Constance 1958; She 1992). The aerial part of $P$. japonicum such as young buds, leaves and berries are used as foodstuff whereas the underground roots have been used as oriental herbal medicine in the treatment of headache, anemia, apoplexy, paralysis, common cold and respiratory illnesses (Amano 1987; Hisamoto et al. 2003; Kang et al. 2013). P. japonicum has a variety of utilities. It is commonly used as a leafy vegetable and an herbal medicine, and a growing interest in this plant has increased farmer's cultivation in Korea. However, despite this growing interest, genomic information to advance breeding efforts on $P$. japonicum is limited (Seo et al. 2001; Han et al. 2017).

Next-generation sequencing (NGS) technology is widely used because of its high-throughput productivity (Varshney et al. 2009; Choi et al. 2011; Kim 2012). Recently, we developed a high-throughput method to assemble complete sequences of chloroplast genome and nuclear ribosomal DNA (nrDNA) simultaneously using low coverage WGS data, coined as de novo assembly using low coverage WGS (dnaLCW) (Kim et al. 2015b). We applied the dnaLCW method very efficiently to finding inter- or intra-species polymorphism and developing markers to differentiate species or cultivars based on the completely assembled chloroplast and nrDNA sequences in diverse plant species (Kim et al. 2015a). The dnaLCW method was successfully applied to develop markers for genetic diversity for resource plants. However, the information is limited to the chloroplast genome and nrDNA which are highly conserved genomic regions. Therefore, large-scale SSR markers derived from nuclear genomes are necessary for further genetics and genomics approach for breeding. Here, we would like to expand our approach to find large-scale polymorphic SSR markers using WGS data and provide useful genome information and genetic pSSR markers that can be utilized for genetics and breeding of the valuable resource plant, $P$. japonicum.

\section{MATERIALS AND METHODS}

\section{Plant materials, genomic DNA extraction and NGS sequencing}

We collected various wild $P$. japonicum collections in the Southern coastal area in Korea. We maintain these collections in Seoul National University farm, Suwon, in Korea. Among them, seven P. japonicum collections were used in this study. Three wild individuals were collected from Jeju Island (hereafter PJ \#1), Geumo Island (hereafter PJ \#2) and Wan Island (hereafter PJ\#3) in Korea. The other two individuals (hereafter PJ\#4 and PJ\#5) were provided from Hantaek botanical garden (http://www.hantaek.co.kr, South Korea) and other two individuals (hereafter PJ\#6 and PJ\#7) were provided from Medicinal herb garden of college of Pharmacy in Seoul National University (http://www.snuherb.ac.kr). Hereafter we will refer all $P$. japonicum sample to their collection number. The fresh leaves of seven $P$. japonicum individuals were collected for DNA preparation. Each leaf sample was grinded separately using liquid nitrogen. The genomic DNAs were extracted using a modified cetyltrimethylammonium bromide (CTAB) method (Allen et al. 2006). Among seven plants, $\mathrm{PJ} \# 1$ and $\mathrm{PJ} \# 2$ were used to construct genomic libraries with insert size of $500 \mathrm{bp}$, according to Illumina paired-end (PE) standard protocol (http://www.illumina. com) and sequenced using Illumina MiSeq genome analyzer from LabGenomics (www.labgenomics.co.kr). The raw sequences of PJ\#1 and PJ\#2 have been uploaded at NABIC database and registered as NN-2130-000001 and NN-2132-000001, respectively (http://nabic.rda.go.kr/) (Seol et al. 2016). 


\section{Sequence preparation (quality control and paired reads joining), identification of SSR motif and clustering}

WGS reads of PJ\#1 and PJ\#2 were trimmed off using Trimmomatic (ver. 0.33) based on quality score and sequence length; condition is set to the minimum quality score of $\geq 20$ and read length of $\geq 70$ bp (Bolger et al. 2014). Trimmed PE reads of PJ\#1 were further assembled for joining of both forward and reverse PE sequences for each read by clc_overlap_reads (ver. 4.21.104315, CLC Inc, Aarhus, Denmark). We assembled forward and reverse sequences for each $\mathrm{PE}$ reads and made one PE-joined contigs (contigs) by combining of both forward and reverse reads based on the overlap. In the contig sets, contigs greater than $250 \mathrm{bp}$ including SSR motifs were identified using microsatellite search module (MISA, http://pgrc.ipk-gatersleben.de/misa/) with the minimum repeat number of $6,5,5,5,5$, and 4 for di-, tri-, tetra-, penta-, hexa-, and hepta-nucleotides, respectively. The contigs of reference species (PJ\#1) were clustered based on homology using BLASTClust (https://www.ncbi.nlm.nih. gov/Web/Newsltr/Spring04/blastlab.html, Altschul et al. 1997) and the clusters with single contigs were selected for further analysis because we considered these sequence as derived from non-repeat genomic regions.

\section{Discovery of pSSRs between two WGS reads}

The WGS reads of PJ\#2 were aligned with the contigs of PJ\#1 in previous step by using clc mapper (ver. 4.21.104315, CLC Inc, Aarhus, Denmark). Afterward, we have located the candidates of pSSR through comparison between contigs of PJ\#1 and WGS reads of PJ\#2 using clc_find_variation (ver. 4.21.104315, CLC Inc, Aarhus,
Denmark). The information of the sequence variation file and the SSR motif file from MISA were combined to estimate the polymorphic regions including microsatellite sites using in-house python program.

\section{PCR validation of SSR markers}

Using sequences information with the candidate pSSR sites, SSR markers were designed by Primer3 (Rozen et al. 2000; Koressaar and Remm 2007; Untergasser et al. 2012). Genomic DNAs of seven $P$. japonicum collections were used as templates for PCR validation of SSR markers. PCR amplification was carried out in a $25-\mu \mathrm{L}$ reaction volume containing the following components: $20 \mathrm{ng}$ of DNA template, $10 \mu \mathrm{M}$ of primer set, $5 \mathrm{mM}$ of dNTP, and one unit Taq DNA polymerase (Vivagen, Seongnam, Korea). For six primer sets, PjSSR01 to PjSSR06, the amplification condition was as follows: 5 minutes at $94^{\circ} \mathrm{C}, 35$ cycles of $94^{\circ} \mathrm{C} 20$ seconds, $58^{\circ} \mathrm{C} 20$ seconds, and $72^{\circ} \mathrm{C} 20$ seconds and then $72^{\circ} \mathrm{C}$ for 7 minutes. For other primer sets, PjSSR07 to PjSSR10, the amplification condition was the same as above except annealing condition of $54^{\circ} \mathrm{C}$ for 20 seconds. PCR products were then separated by $9 \%$ polyacrylamide gel electrophoresis for identification of polymorphisms. Gel was stained with ethidium bromide and visualized under UV lamps for manual genotyping.

\section{RESULTS}

\section{Identification of pSSR using two WGS in $P$. japonicum}

We collected diverse germplasms for $P$. japonicum which is an indigenous plant in Korea. Among the

Table 1. Sequencing status of two $P$. japonicum collections.

\begin{tabular}{lrrrrrr}
\hline \hline & \multicolumn{2}{c}{ P. japonicum } & acc. \#1 (PJ\#1) & & \multicolumn{2}{c}{ P. japonicum acc. \#2 (PJ\#2) } \\
\cline { 2 - 3 } \cline { 5 - 6 } & \# of reads & base pairs & & \# of reads & base pairs \\
\hline Raw data & $3,550,678$ & $1,063,845,547$ & & $3,937,550$ & $1,183,084,443$ \\
Trimmed data & $3,438,470$ & $869,332,349$ & & $3,817,637$ & $978,732,808$ \\
PE joined contig (contigs) & $1,646,551$ & $595,380,248$ & & & \\
SSR containing contigs & 25,814 & & $9,891,314$ & & \\
Primary filtered contigs by clustering & 12,206 & & $4,565,371$ & & \\
Secondary filtered contigs by mapping & 4,698 & & $1,878,413$ & & \\
pSSR containing contigs & 452 & 179,609 & & \\
\hline
\end{tabular}


collections, we carried out WGS sequencing for two individuals, PJ\#1 and PJ\#2, which were collected from Jeju Island and Geumo Island, respectively. Raw WGS of PJ\#1 and PJ\#2 were approximately $1.1 \mathrm{Gbp}$ and $1.2 \mathrm{Gbp}$, respectively. After sequence quality filtering, a total amount of $870 \mathrm{Mb}$ and $980 \mathrm{Mb}$ of WGS data remained for PJ\#1 and PJ\#2, respectively (Table 1). MiSeq sequences usually provide average $300 \mathrm{bp}$ for forward and reverse sequence of each PE read. Our PE library was constructed from $500 \mathrm{bp}$ insert size DNA fragments. To obtain relatively long reads and reduce the redundant finding from same sequences derived from forward and reverse sequence of same PE read, we assembled forward and reverse sequences for each PE reads to make PE-joined contigs (contigs) based on the overlapped sequences. The high quality 3.4 million reads were reduced to $1.6 \mathrm{M}$ contigs by pair joining. Using the contigs, we conducted following steps for in silico identification of pSSRs. First, we selected SSR motif from PJ\#1 through MISA program. Second, considerably non-redundant contigs were chosen as candidates by sequence clustering because high copy reads might be derived from repetitive sequence regions. SSR motifs were identified from 25,814 contigs, of which
12,206 were chosen from the solitary clustered contigs (Table 1). To identify pSSR motifs between PJ\#1 and PJ\#2, WGS reads of PJ\#2 were aligned onto 12,206 contigs of PJ\#1. During alignment, the 1,376 contigs with high mapping depth $(\geq 10)$ and 6,132 contigs without any counterpart mapped reads were excluded. The remaining 4,698 contigs were then compared with SSR motif of PJ\#2 and only 452 contigs contains pSSR motif were selected for further analysis. Among them, 371, 60, 21, and 7 contigs have di-, tri-, tetra- and more than penta-nucleotide SSR motifs, respectively (Fig. 1). Among the 452 pSSR contigs, five contigs had more than 2 SSR motifs (Supplementary Table S1).

\section{Validation of pSSR and application for $P$. japonicum germplasm}

To validate the pSSRs, we randomly chose ten contigs and designed primer pairs to amplify the SSR regions using Primer3 (Table 2). We included five more $P$. japonicum plants (PJ\#3-7) for genotyping each collections using the primer pairs. All ten primer sets (PjSSR01 10) successfully amplified each of $P$. japonicum collections. Nine of ten produced the estimated polymorphic SSR

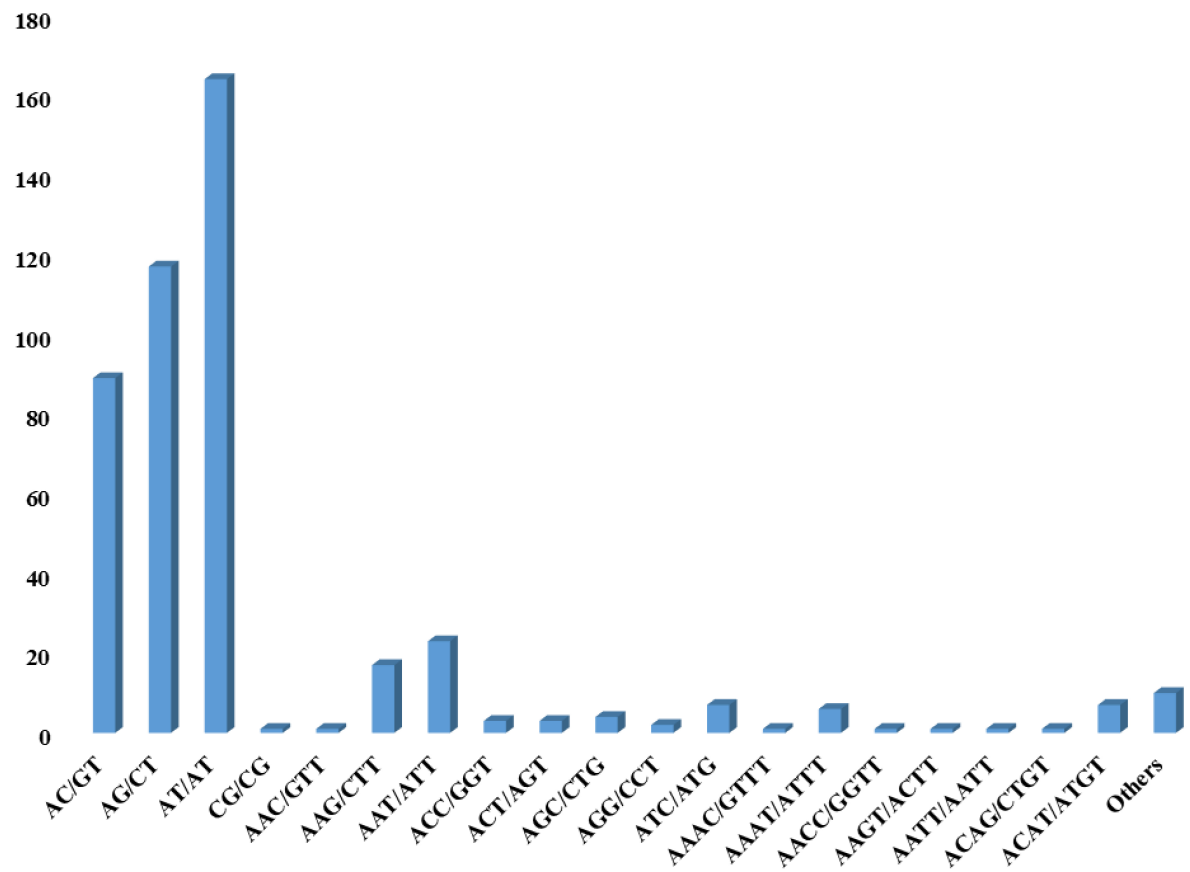

Fig. 1. Classification of pSSR candidates based on the SSR motif in P. japonicum. The horizontal axis represents the type of SSR motif and vertical axis represents the number of SSR motifs. 


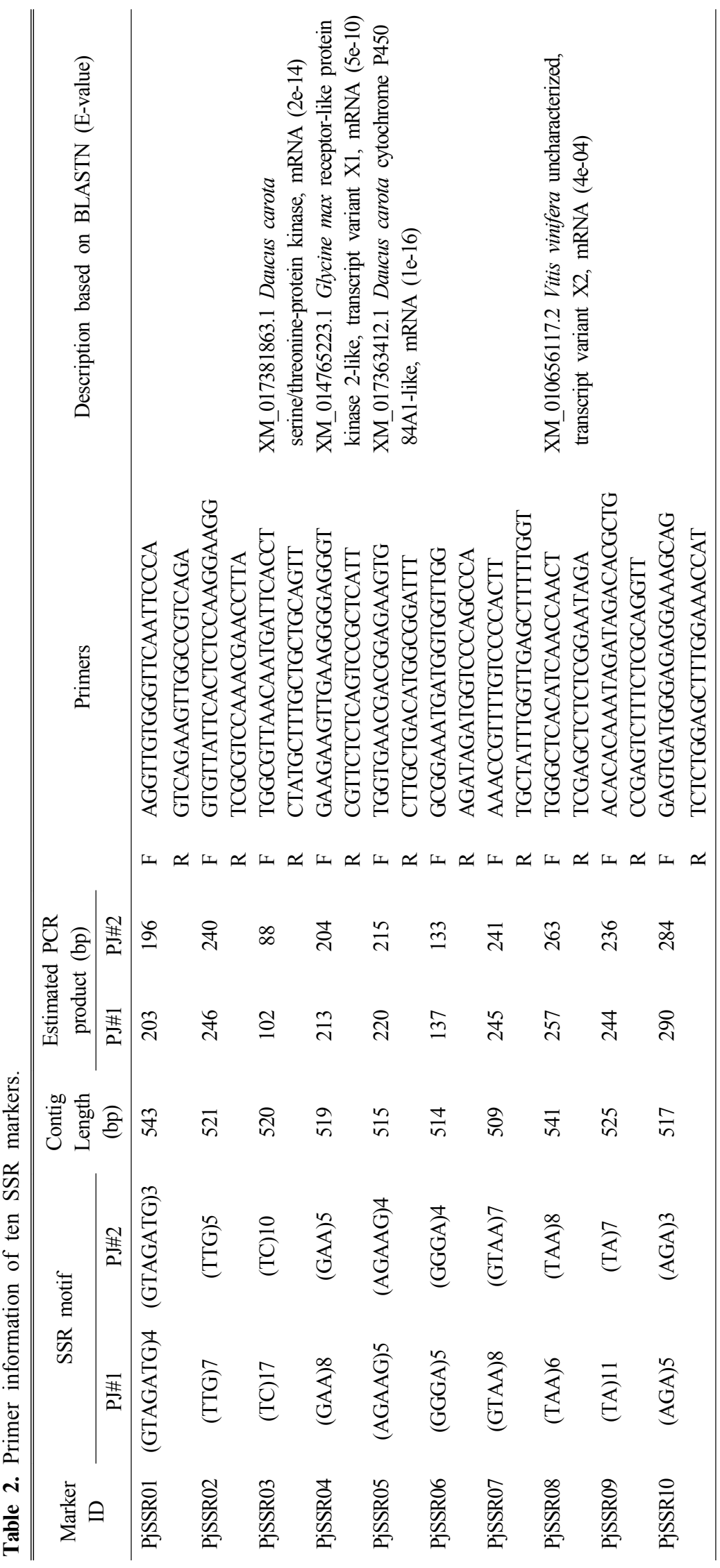


genotypes derived from the copy numbers of SSR units among $P$. japonicum collections (Fig. 2). Meanwhile, one primer, PjSSR08, did not show any polymorphism among genotypes which differ from the expectation (Table 3).
The genotypes of the $P$. japonicum collections for each marker are represented in Table 3 . The mean allele number of developed markers was around 2.7, whereas the mean genetic diversity and polymorphic information contents
A

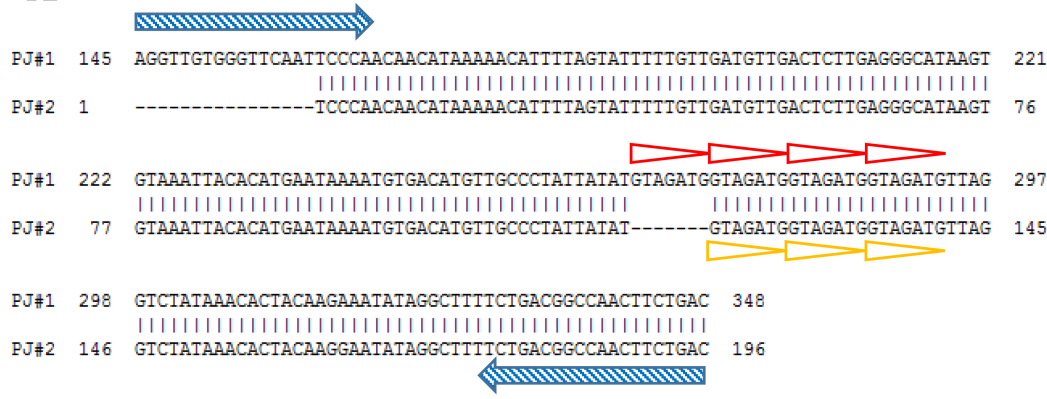

C

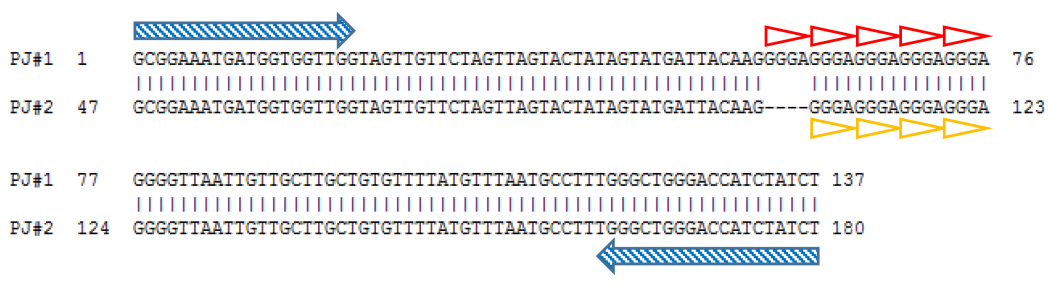

B

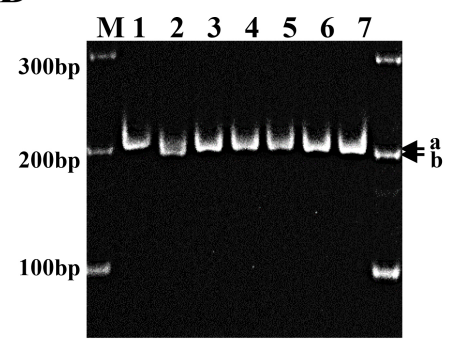

D

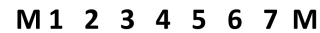

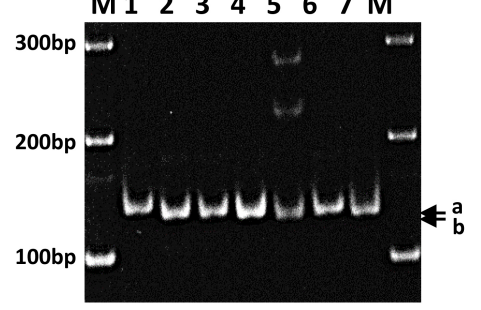

Fig. 2. In silico prediction and PCR validation of PjSSR01 and PjSSR06 primers. (A, C) Sequence alignment between contigs of PJ\#1 and the counterpart WGS reads of PJ\#2 for the primer regions, PjSSR01 and PjSSR06. Blue arrows indicate the forward and reverse primers and the colored triangle boxes in red and yellow represent the SSR motifs for PJ\#1 and PJ\#2, respectively. (B, D) Gel electrophoresis results of PCR products for PjSSR01 and PjSSR06. The number 1 to 7 indicate seven $P$. japonicum collections, PJ\#1 to PJ\#7, respectively. $\mathrm{M}$ indicates 100-bp ladder. Arrows with alphabets $(a, b)$ indicate different alleles among seven collections.

Table 3. Genotypes and allele diversity of 10 markers among seven collections of P. japonicum.

\begin{tabular}{|c|c|c|c|c|c|c|c|c|c|c|}
\hline Primers & PJ\#1 & PJ\#2 & PJ\#3 & PJ\#4 & PJ\#5 & PJ\#6 & PJ\#7 & $\begin{array}{l}\text { No. of } \\
\text { alleles }\end{array}$ & $\begin{array}{c}\text { Genetic } \\
\text { diversity }^{\text {z) }}\end{array}$ & $\mathrm{PIC}^{\mathrm{y})}$ \\
\hline PjSSR01 & $\mathrm{a}$ & $\mathrm{b}$ & $\mathrm{a}$ & $\mathrm{a}$ & $\mathrm{a}$ & $\mathrm{a}$ & $\mathrm{b}$ & 2 & 0.408 & 0.325 \\
\hline PjSSR02 & $\mathrm{a}$ & b & $\mathrm{b}$ & b & b & $b$ & $\mathrm{c}$ & 3 & 0.449 & 0.406 \\
\hline PjSSR03 & $\mathrm{a}$ & $\mathrm{c}$ & b & $\mathrm{c}$ & $\mathrm{c}$ & $\mathrm{c}$ & $\mathrm{c}$ & 3 & 0.449 & 0.406 \\
\hline PjSSR04 & $\mathrm{b}$ & $\mathrm{a}$ & d & $\mathrm{a}$ & $\mathrm{a}$ & $\mathrm{c}$ & $\mathrm{c}$ & 3 & 0.694 & 0.641 \\
\hline PjSSR05 & $\mathrm{a}$ & b & $\mathrm{a}$ & $\mathrm{c}$ & $\mathrm{c}$ & $\mathrm{a}$ & b & 3 & 0.653 & 0.580 \\
\hline PjSSR06 & $\mathrm{a}$ & b & $\mathrm{b}$ & b & b & $\mathrm{a}$ & $\mathrm{a}$ & 2 & 0.490 & 0.370 \\
\hline PjSSR07 & $\mathrm{b}$ & $\mathrm{c}$ & d & $\mathrm{c}$ & $\mathrm{c}$ & $\mathrm{c}$ & $\mathrm{a}$ & 4 & 0.612 & 0.570 \\
\hline PjSSR08 & $\mathrm{a}$ & $\mathrm{a}$ & $\mathrm{a}$ & $\mathrm{a}$ & $\mathrm{a}$ & $\mathrm{a}$ & $\mathrm{a}$ & 1 & 0.000 & 0.000 \\
\hline PjSSR09 & $\mathrm{a}$ & b & $\mathrm{b}$ & b & b & b & $\mathrm{b}$ & 2 & 0.245 & 0.215 \\
\hline PjSSR10 & $\mathrm{a}$ & $\mathrm{a}$ & b & $\mathrm{c}$ & $\mathrm{b}$ & $\mathrm{c}$ & d & 4 & 0.735 & 0.685 \\
\hline Mean & & & & & & & & 2.7 & 0.473 & 0.420 \\
\hline
\end{tabular}

${ }^{\mathrm{z})}$ Genetic diversity is the probability to show difference between two randomly chosen alleles from the population.

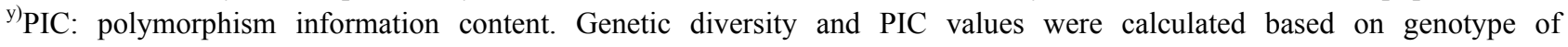
individuals by PowerMarker ver. 3.0 (Liu et al. 2005). 
(PIC) were around 0.47 and 0.42 , respectively. We believe that the pSSR targets will be valuable resources and the ten SSR markers will be beneficial to evaluate and classify $P$. japonicum genotype for genetic study. We carried out blastn analysis (https://blast.ncbi.nlm.nih.gov/Blast.cgi) against non-redundant (nr) nucleotide sequence database and identified that four of ten $P$. japonicum primer regions were similar to genic regions of other plant species (Table 2).

\section{DISCUSSION}

Resource plants have a variety of useful potentials and some resource plants can be developed as minor food crops or pharmaceutical materials. Minor crops are crops with high value but not widely studied, hence it is also known as an 'orphan crops' or 'underutilized crops'. In addition, genomic or genetic information of minor crops is rarely reported. SSR marker is one of the most applicable markers used the genetic evaluation and utilization of crops due to its high-reproducibility, hyper-variable and co-dominant nature (Park et al. 2009). Therefore, many efforts were conducted to develop SSR markers by construction and sequencing of SSR-rich genomic libraries for the minor crops and by utilization of EST sequence (Choi et al. 2011; Kim 2012; Izzah et al. 2014). However, previous researches required the time consuming wet experiments to find polymorphic SSR markers due to low polymorphism rate (with less than $5 \%$ of success rate) from the SSR candidates because they utilized sequence harboring the SSR motif from one genotype (Choi et al. 2011; Kim 2012; Izzah et al. 2014). In this study, we explored two WGSs to discover polymorphic SSR markers for the valuable resource plants $P$. japonicum as leafy vegetable and functional foods. Moreover, we have also confirmed that our designed SSR primer sets could be successfully applied in other collections. Ten SSR primers (PjSSR01 10) were successfully amplified, with high polymorphism in all collections (Table 2 and 3 ). Some primers showed different results from our expectation. The PCR products of both PjSSR04 and PjSSR10 were smaller than expected sizes and PjSSR08 did not show any polymorphism. These unexpected results might be caused by paralogous sequences in P. japonicum. Despite the unexpected results, however, our SSR markers displayed polymorphic amplicons with high success rate ( 9 out of 10). The 452 pSSR candidates sequence will provide fundamental information on evaluation of useful and fundamental genetic resources for breeding in $P$. japonicum (Table 2 and Supplementary Table S1).

\section{ACKNOWLEDGEMENTS}

This research was supported by "Cooperative Research Program for Agriculture Science \& Technology Development (Project Nos. PJ01103001)", Rural Development Administration, Republic of Korea. We thank Hantaek botanical garden and Medicinal herb garden of college of Pharmacy in Seoul National University for providing plant materials for our study.

\section{REFERENCES}

Abdelkrim J, Robertson B, Stanton JA, Gemmell N. 2009. Fast, cost-effective development of species-specific microsatellite markers by genomic sequencing. Biotechniques 46: $185-192$.

Agarwal M, Shrivastava N, Padh H. 2008. Advances in molecular marker techniques and their applications in plant sciences. Plant Cell Rep. 27: 617-631.

Allen G, Flores-Vergara M, Krasynanski S, Kumar S, Thompson W. 2006. A modified protocol for rapid DNA isolation from plant tissues using cetyltrimethylammonium bromide. Nat. Protoc. 1: 2320-2325.

Altschul SF, Madden TL, Schaffer AA, Zhang J, Zhang Z, Miller W, et al. 1997. Gapped BLAST and PSI-BLAST: a new generation of protein database search programs. Nucleic Acids Res. 25: 3389-3402.

Amano T. 1987. Ryukyuretto syokubutu hougensyu (The botanical dialects in the Ryukyus). Shinseitosho Shuppan: Naha

Buwalda P. 1949. Umbelliferae In: CGGJ van Steenis (ed). Flora Malesiana ser. I 4: 112-140.

Bolger AM, Lohse M, Usadel B. 2014. Trimmomatic: a 
flexible trimmer for Illumina sequence data. Bioinformatics 30: 2114-2120.

Choi H-I, Kim NH, Kim JH, Choi BS, Ahn I-O, Lee J-S, et al. 2011. Development of reproducible EST-derived SSR markers and assessment of genetic diversity in Panax ginseng cultivars and related species. J. Ginseng Res. 35: 399-412.

da Maia LC, Palmieri DA, de Souza VQ, Kopp MM, de Carvalho FI, Costa de Oliveira A. 2008. SSR Locator: tool for simple sequence repeat discovery integrated with primer design and PCR simulation. Int. J. Plant Genomics 2008: 412696.

Grover A, Sharma PC. 2016. Development and use of molecular markers: past and present. Crit. Rev. Biotechnol. 36: 290-302.

Han B-X, Yuan Y, Huang L-Q, Zhao Q, Tan L-L, Song X-W, et al. 2017. Specific PCR identification between Peucedanum praeruptorum and Angelica decursiva and identification between them and adulterant using DNA barcode. Pharmacognosy Magazine 13: 38.

Hiroe M, Constance L. 1958. Umbelliferae of Japan. Univ. of Calif. Bull. Bot. 30:1-444.

Hisamoto M, Kikuzaki H, Ohigashi H, Nakatani N. 2003. Antioxidant compounds from the leaves of Peucedanum japonicum Thunb. J. Agric. Food Chem. 51: 5255-5261.

Izzah NK, Lee J, Jayakodi M, Perumal S, Jin M, Park BS, et al. 2014. Transcriptome sequencing of two parental lines of cabbage (Brassica oleracea L. var. capitata L.) and construction of an EST-based genetic map. BMC Genomics 15: 149.

Jones AG, Small CM, Paczolt KA, Ratterman NL. 2010. A practical guide to methods of parentage analysis. Mol. Ecol. Resour. 10: 6-30.

Kang SY, Oh TW, Kim JW, Park Y-K. 2013. Effect of the water extract of Peucedani Japonici Radix on ovalbumininduced allergic asthma in mice. The Korea Journal of Herbology 28: 1-7.

Kelkar YD, Strubczewski N, Hile SE, Chiaromonte F, Eckert KA, Makova KD. 2010. What is a microsatellite: a computational and experimental definition based upon repeat mutational behavior at $\mathrm{A} / \mathrm{T}$ and $\mathrm{GT} / \mathrm{AC}$ repeats. Genome Biol. Evol. 2: 620-635.

Kim K, Lee SC, Lee J, Lee HO, Joh HJ, Kim NH, et al. 2015 a. Comprehensive survey of genetic diversity in chloroplast genomes and $45 \mathrm{~S}$ nrDNAs within Panax ginseng
Species. PLoS One 10: e0117159.

Kim K, Lee SC, Lee J, Yu Y, Yang K, Choi BS, et al. 2015 b. Complete chloroplast and ribosomal sequences for 30 accessions elucidate evolution of Oryza AA genome species. Sci. Rep. 5: 15655.

Kim NH, Choi HI, Ahn IO, Yang TJ. 2012. EST-SSR marker sets for practical authentication of all nine registered ginseng cultivars in Korea. J. Ginseng Res. 36: 298-307.

Koressaar T, Remm M. 2007. Enhancements and modifications of primer design program Primer3. Bioinformatics 23: 1289-1291.

Liu K, Muse SV. 2005. PowerMarker: an integrated analysis environment for genetic marker analysis. Bioinformatics 21: 2128-2129.

Ma K-H, Kim N-S, Lee G-A, Lee S-Y, Lee JK, Yi JY, et al. 2009. Development of SSR markers for studies of diversity in the genus Fagopyrum. Theor. Appl. Genet. 119: 1247-1254.

Meng W, Fei X, Peng Y, Duan X-Y, Zhou Y-L, Shen C-Y, et al. 2014. Development of SSR markers for a phytopathogenic fungus, Blumeria graminis f. sp. tritici, using a FIASCO protocol. J. Integr. Agric. 13: 100-104.

Metz S, Cabrera JM, Rueda E, Giri F, Amavet P. 2016. FullSSR: microsatellite finder and primer designer. Adv. Bioinformatics 2016:

Mittal N, Dubey AK. 2009. Microsatellite markers-A new practice of DNA based markers in molecular genetics. Pharmacogn. Rev. 3: 235.

Park Y-J, Lee JK, Kim N-S. 2009. Simple sequence repeat polymorphisms (SSRPs) for evaluation of molecular diversity and germplasm classification of minor crops. Molecules 14: 4546-4569.

Rozen S, Skaletsky H. 2000. Primer3 on the WWW for general users and for biologist programmers. Methods Mol. Biol. 132: 365-386.

Seo A, Watanabe M, Hotta M, Murakami N. 2001. Allozyme variation of the three varieties of Peucedanum japonicum Thunb. in Japan. APG: Acta Phytotax. Geobot. 52: 135-148.

Seol YJ, Lee TH, Park DS, Kim CK. 2016. NABIC: a new access portal to search, visualize, and share agricultural genomics data. Evol. Bioinform. Online 12: 51-58.

She M. 1992. Umbellifera. In: S Kenhwa, S Menglan (eds). Fl. Repub. Popul. Sinecae 55: 123-175 (in Chinese)

Untergasser A, Cutcutache I, Koressaar T, Ye J, Faircloth BC, 
Remm M, et al. 2012. Primer3--new capabilities and interfaces. Nucleic Acids Res. 40: e115.

Varshney RK, Graner A, Sorrells ME. 2005. Genic microsatellite markers in plants: features and applications. Trends Biotechnol. 23: 48-55.
Varshney RK, Nayak SN, May GD, Jackson SA. 2009. Next-generation sequencing technologies and their implications for crop genetics and breeding. Trends Biotechnol. 27: 522-530. 